\title{
Response to "Letter to the Editor: Comments on a Recent Article by Burgos et al."
}

\author{
J. M. Burgos, B. A. Ellington, and M. F. Varela ${ }^{1}$ \\ Eastern New Mexico University, Department of Biology, Station 33, Portales 88130
}

We wish to respond to the letter by Shryock et al. (2005) regarding our paper (Burgos et al., 2005). Their comments were, regrettably, inaccurate. First, Shryock and colleagues contended that we tested chloramphenicol, nalidixic acid, tetracycline, and penicillin because of their widespread use in dairy cattle. This is obviously incorrect, as some of these agents most certainly are not used in dairy cattle. What we did state was, "Four antibiotics were chosen (note new emphasis here) either because of their association with mar or their widespread use in dairy cattle" (Burgos et al., 2005). This clearly has a different meaning than that attributed by Shryock et al. (2005). Readers will note that bacterial resistance to chloramphenicol, nalidixic acid, and penicillin is increased when the mar locus is induced (Randall and Woodward, 2002), which was the deciding factor in choosing the antimicrobial agents for study. We agree with Shryock et al. (2005) about the agricultural use of tetracycline. In fact, tetracycline is commonly known to be extensively used as a growth enhancer in calves (Levy, 2002). This is why we chose to study tetracycline.

Second, Shryock et al. (2005) stated that we employed nonstandard methods for the determination of MIC. This is again inaccurate. We wish to resolve this confusion by pointing out, for the benefit of the reader, that 1 ) a multitude of methods for MIC determinations exist (gradient plate, use dilution, E-test strip, Kirby-Bauer, disc diffusion, spiral, enzymatic, etc.), and 2) each of these methods is associated with its own protocol that is standardized, approved by the National Committee for Clinical Laboratory Standards, established, published, and routinely used by investigators. Therefore, it is reasonable to maintain that there is general acceptability of our gradient plate method (Koutsolioutsou et al., 2005) as well. As stated in our paper, we invoked the National Committee for Clinical Laboratory Standards (2002) method for MIC determination of aerobic bacteria from the Enterobacteriaceae family. The standardized protocols for the gradient plate method that we employed

Received November 18, 2005.

Accepted December 19, 2005.

${ }^{1}$ Corresponding author: Manuel.Varela@enmu.edu are not only approved by the National Committee for Clinical Laboratory Standards, which includes standard interpretive specifications, but are also used widely and accepted in the nonclinical arena (Curiale and Levy, 1982; George and Levy, 1983; Hachler et al., 1991; Carsenti-Dellamonica et al., 2005; Koutsolioutsou et al., 2005). Our isolates were not clinical isolates, but rather were soil isolates. As such, our isolates were not shown to be causative agents of infectious diseases. Thus, a clinical method for MIC determination was not indicated in our study. With respect to the potential pathogenicity of the isolates, however, it seems to us that simply having the reservoir of resistant organisms is highly relevant, whether they are infectious or not, because transfer of plasmids from nonpathogenic or nonvirulent strains to their pathogenic or virulent counterparts is widely documented.

\section{REFERENCES}

Burgos, J. M., B. A. Ellington, and M. F. Varela. 2005. Presence of multidrug resistant enteric bacteria in dairy farm topsoil. J. Dairy Sci. 88:1391-1398.

Carsenti-Dellamonica, H., M. Galimand, F. Vandenbos, C. Pradier, P. M. Roger, B. Dunais, M. Sabah, G. Mancini, and P. Dellamonica. 2005. In vitro selection of mutants of Streptococcus pneumoniae resistant to macrolides and linezolid: Relationship with susceptibility to penicillin $\mathrm{G}$ or macrolides. J. Antimicrob. Chemother. 56:633-642.

Curiale, M. S., and S. B. Levy. 1982. Two complementation groups mediate tetracycline resistance determined by Tn10. J. Bacteriol. 151:209-215.

George, A. M., and S. B. Levy. 1983. Amplifiable resistance to tetracycline, chloramphenicol, and other antibiotics in Escherichia coli: Involvement of a non-plasmid-determined efflux of tetracycline. J. Bacteriol. 155:531-540.

Hachler, H., S. P. Cohen., and S. B. Levy. 1991. marA, a regulated locus which ontrols expression of chromosomal multiple antibiotic resistance in Escherichia coli. J. Bacteriol. 173:5532-5538.

Koutsolioutsou, A., S. Pena-Llopis, and B. Demple. 2005. Constitutive soxR mutations contribute to multiple-antibiotic resistance in clinical Escherichia coli isolates. Antimicrob. Agents Chemother. 49:2746-2752

Levy, S. B. 2002. Factors impacting on the problem of antibiotic resistance. J. Antimicrob. Chemother. 49:25-30.

National Committee for Clinical Laboratory Standards (NCCLS). 2000. Approved standard M7-A5. In Methods for Dilution Antimicrobial Susceptibility Tests for Bacteria that Grow Aerobically, 5th ed., vol. 20, no. 2. NCCLS, Wayne, PA.

Randall, L., and M. J. Woodward. 2002. The multiple antibiotic resistance (mar) locus and its significance. Res. Vet. Sci. 72:87-93.

Shryock, T. R., J. L. Watts, and J. T. Gray. 2005. Letter to the Editor: Comments on a recent article by Burgos et al. J. Dairy Sci. 88:4136. 